\title{
Very Late-Onset Systemic Lupus Erythematosus as Unusual Cause of Reversible Functional and Cognitive Impairments in an Octogenarian Patient
}

\author{
$\underline{S t e ́ p h a n i e ~ d e ~ M o n t j o y e ~}^{1}$, Benoit Boland ${ }^{2}$, Jacqueline Van Raemdonck ${ }^{2}$, Frédéric A Houssiau ${ }^{1}$ \\ ${ }^{1}$ Rheumatology Department, Cliniques Universitaires Saint-Luc, Université Catholique de Louvain, Brussels, Belgium \\ ${ }^{2}$ Geriatric Medicine Department, Cliniques Universitaires Saint-Luc, Université Catholique de Louvain, Brussels, Belgium
}

\section{Doi: 10.12890/2020_001570 - European Journal of Case Reports in Internal Medicine - @ EFIM 2020}

Received: $11 / 02 / 2020$

Accepted: $25 / 02 / 2020$

Published: 07/05/2020

\begin{abstract}
How to cite this article: de Montjoye S, Boland B, Van Raemdonck J, Houssiau FA. Very late-onset systemic lupus erythematosus as unusual cause of reversible functional and cognitive impairments in an octogenarian patient. EJCRIM 2020;7: doi:10.12890/2020_001533.
\end{abstract}

Conflicts of Interests: The Authors declare that there are no competing interests.

This article is licensed under a Commons Attribution Non-Commercial 4.0 License

\section{ABSTRACT}

While functional decline is a common syndrome in geriatric medicine, the diagnosis of the underlying disease can be complex. We present a case of very late-onset systemic lupus erythematosus with fever, arthritis, lymphadenopathy, sicca syndrome, pleurisy, renal impairment and reversible functional and cognitive impairments. Prompt improvement was observed on prednisolone and hydroxychloroquine.

\section{LEARNING POINTS}

- Systemic lupus erythematosus (SLE) rarely occurs in octogenarian patients.

- In such oldest old patients, SLE may predominantly present with subacute cognitive and functional impairments.

- Low-dose treatment (prednisolone $7.5 \mathrm{mg} /$ day and hydroxychloroquine $5 \mathrm{mg} / \mathrm{kg} /$ day) can reverse all SLE manifestations within 1 month.

\section{KEYWORDS}

Late-onset systemic lupus erythematosus, cognitive impairment, functional decline

\section{INTRODUCTION}

While functional decline is very common in geriatric medicine, the diagnosis of an underlying disease can be complex, more so as the clinical and biological manifestations of the disease may differ in geriatric patients compared to younger patients, making the diagnosis even more difficult. This complexity can be further increased by comorbidities and polymedication. Yet, for a treatable condition, appropriate treatment can be life-saving.

\section{CASE DESCRIPTION}

An 81-year-old Albanian woman was hospitalized for subacute functional impairment, abnormal behaviour, dyspnoea, weight loss (4 kg in 3 months), fever (peak of $38.3^{\circ} \mathrm{C}$ ) and arthralgias. She had suffered from pulmonary tuberculosis and anthracosilicosis in the past. Her active diseases included diabetes mellitus, hypertension and chronic obstructive pulmonary disease. She was being treated with amlodipine, lisinopril, metformin, omeprazole, trazodone and bronchodilators.

Upon admission, clinical examination showed metacarpophalangeal and proximal interphalangeal arthritis. Further examination revealed facial rash, dry eyes and mouth. The neurologic examination was normal, except for impaired spatial and time orientation. The Mini-Mental State Examination score was $7 / 23$ (without the mathematical component as the patient never learned mathematics). The patient was recently unable to write her name or use her mobile phone. 
Blood tests revealed raised C-reactive protein levels at $84 \mathrm{mg} / \mathrm{l}$ and a mild normocytic anaemia. Protein electrophoresis testing showed hypoalbuminaemia $(24 \mathrm{~g} / \mathrm{l}$ ) and mild hypergammaglobulinaemia ( $16 \mathrm{~g} / \mathrm{l})$. Liver and renal function (creatinine $0.5 \mathrm{mg} / \mathrm{dl}$ ) was normal, as were standard coagulation tests. Urinalysis was normal and proteinuria was $0.6 \mathrm{~g} / \mathrm{day}$.

The patient's hand, wrist and knee x-rays showed osteoarthritis. Transthoracic cardiac ultrasound did not show pericardial effusion. Brain magnetic resonance imaging revealed mild leucoaraiosis and spinal fluid examination was normal. 18F-FDG positron emission tomography showed supra- and infra-diaphragmatic lymphadenopathy and splenomegaly, and no evidence of giant cell arteritis. CT angiography revealed a right lower lobe segmental pulmonary embolism, a bilateral pleural effusion and right upper lobe scaring lesions compatible with the patient's past history of tuberculosis.

Testing for antinuclear antibodies was positive (1/160) with high titres of anti-dsDNA antibodies (2,103 U/ml; Farr assay) and anti-SSA antibodies (>240 U/ml; ELISA). Lupus anticoagulant, anti- $\beta 2$-glycoprotein 1 and anti-cardiolipin testing was negative. Serum complement C3/C4 levels were normal.

A diagnosis of systemic lupus erythematosus (SLE) was made. The patient was treated with low-dose prednisolone (7.5 mg/day) and hydroxychloroquine (5 mg/kg/day). To prevent osteoporosis, we prescribed calcium salts and vitamin D3 supplements. Apixaban was started for the pulmonary embolism. The patient improved within 1 week. The arthritis, anorexia and fever disappeared. Cognitive testing improved (she could follow simple orders and use her mobile phone). One month later, anti-dsDNA antibodies had decreased to $855 \mathrm{U} / \mathrm{ml}$, while C-reactive protein levels were $29 \mathrm{mg} / \mathrm{l}$. Urine tests revealed the disappearance of proteinuria. The patient's global health status was back to normal.

\section{DISCUSSION}

SLE is a rare pleiotropic autoimmune disease with an estimated incidence of 5/100,000, a prevalence of $97 / 100,000^{[1]}$ and a peak age of onset of $30 \mathrm{y}$. Late-onset SLE (>50 y) and juvenile-onset SLE (<18 y) correspond to $3-18 \%$ of all cases ${ }^{[2]}$. In late-onset SLE, the female/male ratio is lower ${ }^{[2]}$. Older patients display more sicca syndrome and serositis, although this is not confirmed in all studies ${ }^{[2]}$. Malar rash, photosensitivity, alopecia, cutaneous vasculitis, Raynaud's phenomenon, lymphadenopathy, lymphopenia and lupus nephritis are more common in juvenileonset and adult-onset SLE ${ }^{[2,3]}$. As far as serology is concerned, rheumatoid factor and anti-SSA/SSB antibodies are more commonly detected in late-onset SLE, while lower rates of anti-dsDNA positivity, of anti-Sm positivity and hypocomplementaemia are reported ${ }^{[2]}$.

Rarely, in geriatric patients, lupus-like diseases have been described that are linked to the use of certain drugs, such as procainamide ${ }^{[2]}$. In these cases, labelled "drug-induced lupus", renal involvement is virtually never observed, nor is the presence of anti-dsDNA antibodies.

Hydroxychloroquine, prescribed at a dose of $5 \mathrm{mg} / \mathrm{kg} / \mathrm{day}$, is the first-line treatment for SLE. The drug prevents relapses ${ }^{[2]}$ and improves long-term survival ${ }^{[3,4]}$. Regular eye examination is mandatory, especially in geriatric patients at risk for retinopathy ${ }^{[4]}$. Although glucocorticosteroids remain a standard treatment allowing prompt control of lupus symptoms, we recommend prescribing the lowest possible dose for older patients (7.5-15 mg prednisolone/day) for the shortest possible period of time, as a bridge therapy until the full clinical effect of the antimalarials becomes established. The adverse effects of glucocorticosteroids are indeed especially frequent and severe in geriatric patients ${ }^{[2]}$. Prevention of glucocorticoid-induced bone loss must take place. Exercise must be prescribed to avoid proximal myopathy. Patients should be vaccinated against influenza and Streptococcus pneumoniae.

If the disease is not controlled by the combination of low-dose prednisolone and antimalarials, methotrexate or azathioprine can be prescribed $^{[2]}$, although little data are available on the use of immunosuppressants prescribed for autoimmune diseases in geriatry ${ }^{[5]}$. This treatment must be chosen on a case-by-case basis.

\section{CONCLUSION}

Late-onset SLE is a rare cause of reversible functional and cognitive decline in the elderly.

\section{REFERENCES}

1. Stojan G, Petri M. Epidemiology of systemic lupus erythematosus: an update. Curr Opin Rheumatol 2018;30(2):144-150.

2. Arnaud L, Mathian A, Boddaert J, Amoura Z. Late-onset systemic lupus erythematosus. Drugs Aging 2012;29(3):181-189.

3. das Chagas Medeiros MM, Bezzera MC, Braga FN, da Justa Feijao MR, Gois AC, Rebouças VC, et al. Clinical and immunological aspects and outcome of a Brazilian cohort of 414 patients with systemic lupus erythematosus (SLE): comparison between childhood-onset, adult-onset, and late-onset SLE. Lupus 2016;25(4):355-363.

4. Cartella S, Cavazzana I, Ceribelli A, Inverardi F, Tincani A, Franceschini F. Evaluation of mortality, disease activity, treatment, clinical and immunological features of adult and late onset systemic Lupus erythematosus. Autoimmunity 2013;46(6):363-368.

5. Lahaye C, Tatar Z, Dubost J, Soubrier M. Overview of biologic treatments in the elderly. Joint Bone Spine 2015;82(3):154-160. 